\title{
Identifying Deep Regression Models for Time Series Prediction of Continuous Data in an Anthropomorphic Robot Telecontrol System
}

\author{
G. Edwar Jacinto, Fredy H. Martínez S* and S. Fernando Martínez \\ Facultad Tecnológica, Universidad Distrital Francisco José de Caldas, Bogota D.C., Colombia; \\ ejacintog@udistrital.edu.co,fhmartinezs@udistrital.edu.co,fmartinezs@udistrital.edu.co
}

\begin{abstract}
Objectives: To construct regression models in order to predict the behavior of data produced by motion sensors and muscle activity sensors linked to a human arm, with the intention of coordinating the movement of a robot. Methods: We use a LSTM (Long Short-Term Memory) network setup as a transfer learning problem where a sequence vector is generated and then a probability distribution and a set of real values are output and trained with separate cost functions. Findings: Evaluations are based on the precision accuracy of the algorithms applied to the data provided by the Myo Gesture Control Armband (Thalmic Labs) sensor used by different people with equivalent arm movements. These evaluations show a predictable behavior of the movements independently of the user. Novelty: The use of deep learning to solve a difficult problem of predicting time series avoiding overfitting.
\end{abstract}

Keywords: Data Models, Deep Learning, Myoelectric Signal, Predictive Models, Real-Time, Regression Analysis, Time Series

\section{Introduction}

Mining, filtering and prediction are currently important areas of research needed for time series processing. Time series are a type of temporal data whose value is strongly dependent on historical behavior. The processing of this information is important due to the large amount of data they normally have, which makes their knowledge and identification essential 1 . In addition, the monitoring and study of these time series has become a key element in event-based control schemes $\frac{2,3}{3}$. In a system, an event may be indicative of the need for a specific action by the control unit ${ }^{4}$. In fact, a control system can be completely specified from events in the system ${ }^{5}$. The prediction of events is always a fundamental element in the identification of characteristics of a time series, and therefore an important topic in research.

The processing of the time series consists in finding models for the data that allow discovering hidden knowledge in them, unknown patterns that can be used in a determined action ${ }^{6}$. The data set is particularly special, since it is data collected over time. The objective of the analysis may be:

- Prediction,

- Description and

- Control.

Among the most relevant problems of current research are the high dimensionality of the data and the noise present in them. In the first case, it is sought to construct behavior models that allow the identification of a relevant subset of data, which is reached by eliminating as much irrelevant and redundant data as possible. Some algorithms used include the identification of clusters from features $\frac{7.8}{}$ and dimensional reduction schemes ${ }^{9,10}$.

In terms of noise reduction, some strategies used include the use of the f-transform to reduce the variability of random noise $\frac{11,12}{}$ and Singular Spectrum Analysis (SSA) is used as a noise data classifier ${ }^{13,14}$.

As for the different existing techniques, these can be classified into two categories: Estimator of short-lived

${ }^{*}$ Author for correspondence 
and estimator of long-range ${ }^{6}$. Estimator of short-lived is an estimator of one-ahead-step or short-term and forecast several minutes, hours, days or months ahead ${ }^{15}$. An estimator of long-range on the other hand, is a tool that allows taking long-term decisions ${ }^{16}$. Among the tools used in both cases we can highlight the use of temporary association rules ${ }^{17}$ and neural networks ${ }^{18}$.

This paper is part of a research that seeks the remote control of an anthropomorphic robot. We want to control dynamically, imitating the movement of a human arm on which motion sensors have been placed. This is a first step for the development of non-invasive human prostheses.

We are using a Myo Gesture Control Armband (Thalmic Labs) sensor, which supplies a raw sensor data. The sensor provides EMG (Electromyography) signals (an eight element array, one for each sensor, at $200 \mathrm{~Hz}$ ) which at first sight are quite different from one person to another. The purpose is to propose a model of prediction and behavior of these signals from deep learning to simplify the control of the robot.

We propose the use of a LSTM (Long Short-Term Memory) with one output target, a softmax output and a real numbered vector output. The LSTM is a Recurrent Neural Network (RNN) architecture that unlike traditional neural networks has a high capacity to learn from experience to classify, process and predict time series when there are time lags of unknown size and bound between important events. The insensitivity to gap length gives an advantage to LSTM over alternative RNNs and hidden Markov models and other sequence learning methods in numerous applications.

The following part of the paper is arranged in this way. Section 2 presents preliminary concepts and problem formulation. Section 3 illustrates the methodology based on LSTM to look for patterns in the data. Section 4 we present the preliminary results. And finally, in Section 5, we present our conclusions.

\section{Problem Formulation}

The Myo armband provides data of eight surface EMG (Electromyography) sensors and a 9-axis IMU (Inertial Measurement Unit) module. The Myo armband outputs raw data from the 9-axis IMU includes:

- Raw accelerometer data.

- Raw gyroscope data.

- Orientation data.
The EMG data is at $200 \mathrm{~Hz}$ and the IMU data is at $50 \mathrm{~Hz}$. In this research we are working with data from the eight EMG channels. EMG sensors are located around the user's arm, each supplying an 8-bit data channel. We recorded the data of the sensors for a gesture (clenching the fist) in three different people:

- User 1: Male, ectomorph body, little hair on the arm, 20 years old Figure 1.

- User 2: Woman, endomorph body, moderate hair in the arm, 21 years old Figure 2.

- User 3: Male, endomorph body, little hair on the arm, 22 years old Figure 3.

The three individuals used the Myo armband for the first time during the test. The data were transmitted wirelessly by bluetooth to a computer for recording and analysis.

We need to analyze these data because the structure and physiology of the muscles varies from person to person and even over time. Even more, the EMG readings can be altered by factors such as arm circumference, forearm strength, arm hair and body fat. At first sight, it is not possible to derive from the data a theoretical model that explains the behavior of the signals, which is why it is proposed to construct a regression model in order to predict the behavior, in this case using a LSTM (Long Short-Term Memory). LSTM is a Recurrent Neural Network (RNN) architecture that unlike traditional neural networks has a high capacity to learn from experience to classify process and predict time series when there are time lags of unknown size and bound between important events.

Recurrent Neural Networks have feedback loops that allow them to maintain information about past events (memory) over a certain period of time. These networks transform an input sequence into an output sequence by considering the information in both sequences. The training of these networks is more complex than the training of traditional static networks and it is well known that a suboptimal solution takes into account short-term dependencies but not long-term dependencies. LSTMs are explicitly designed to avoid the long-term dependency problem.

\section{Methodology}

Figures 1, 2 and 3 show a predictable pattern on all channels independent of the user. It is possible to observe a 

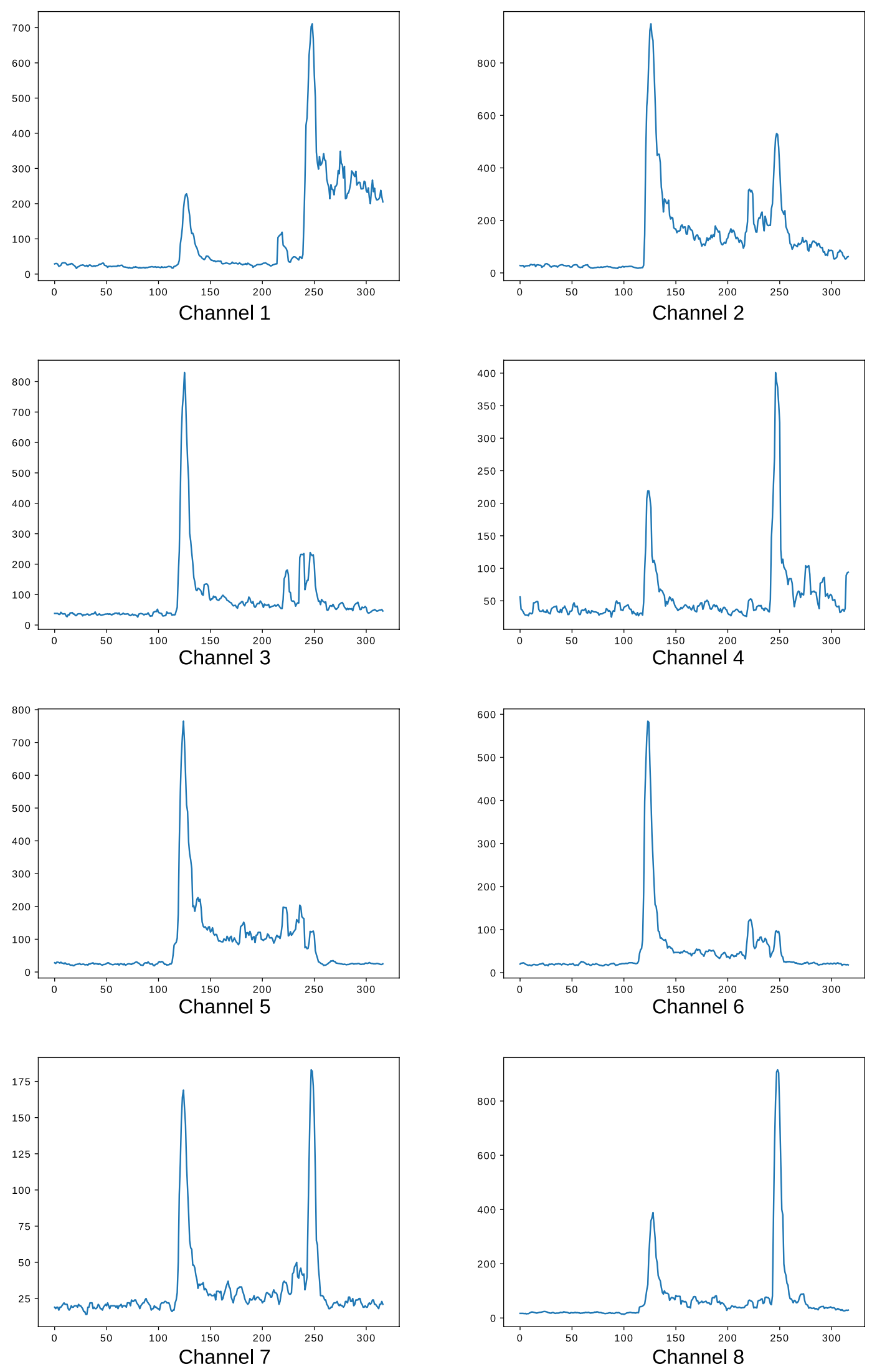

Figure 1. Eight EMG signals for User 1 (Male, ectomorph body, little hair on the arm, 20 years old). 

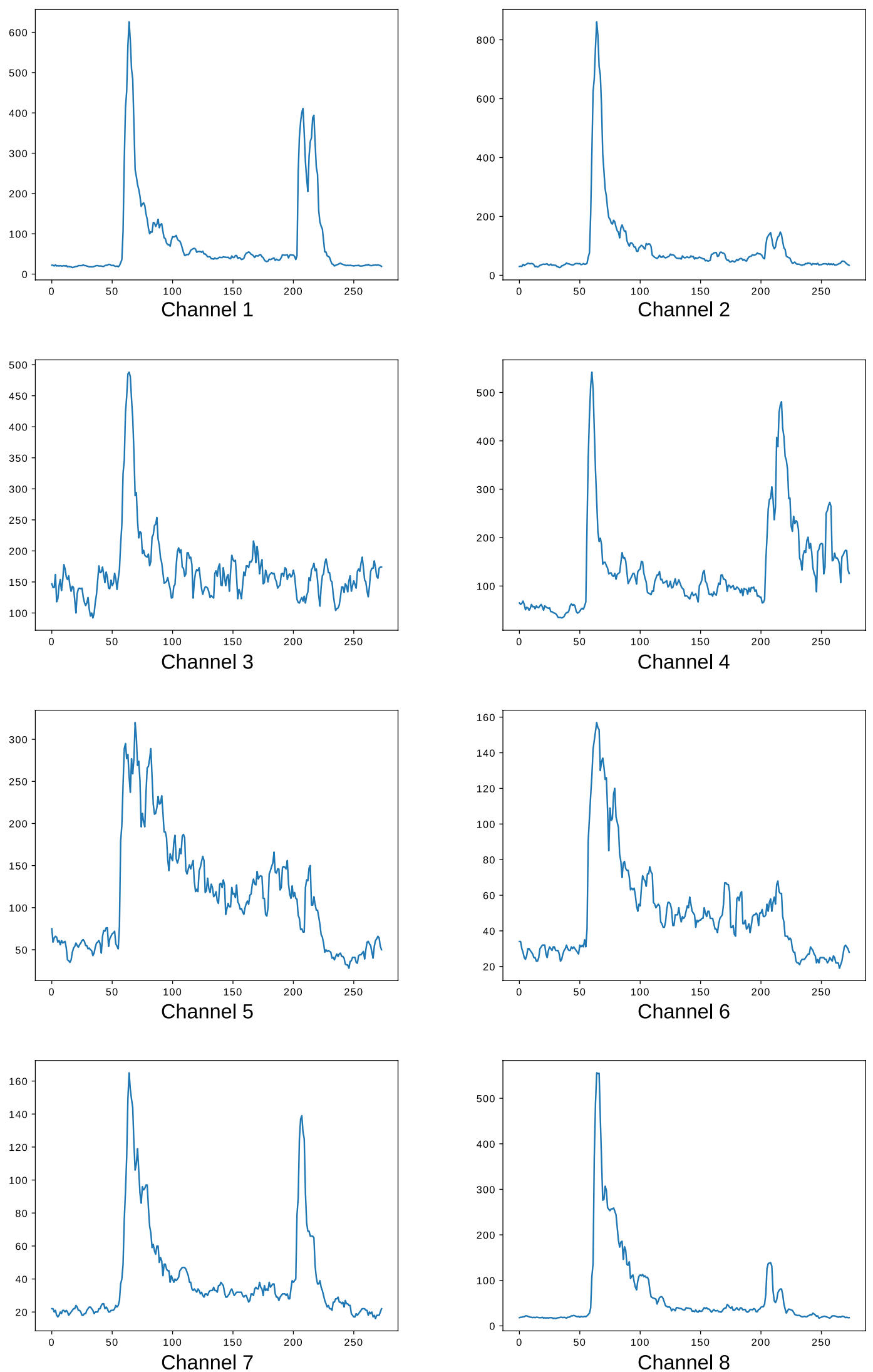

Figure 2. Eight EMG signals for User 2 (Woman, endomorph body, moderate hair in the arm, 21 years old). 

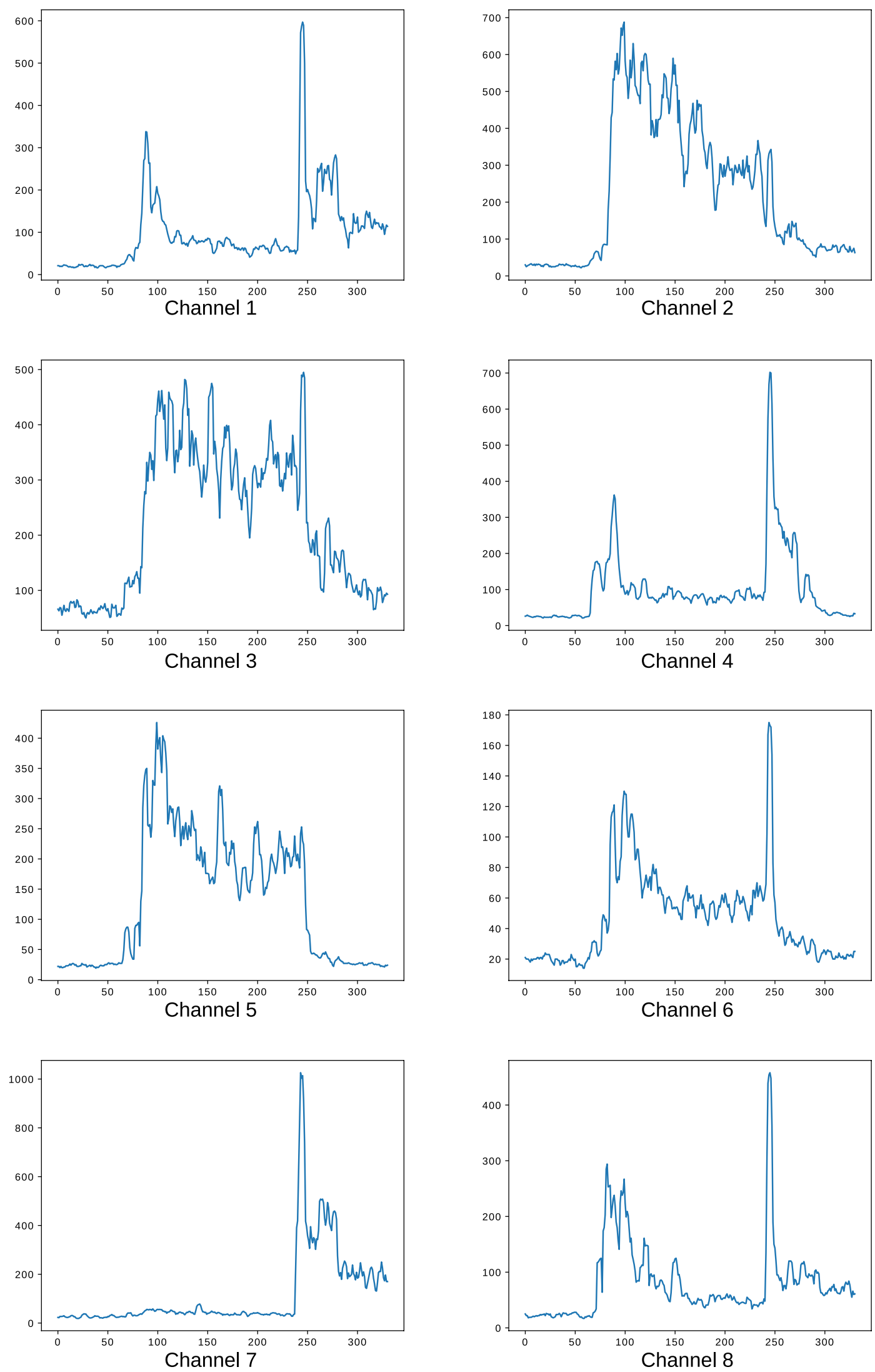

Figure 3. Eight EMG signals for User 3 (Male, endomorph body, little hair on the arm, 22 years old). 
valley and two peaks in the data. To estimate the behavioral model of these data we use an LSTM. LSTM networks use memory blocks instead of neurons. The blocks have gates that handle both the state of the block and its output. These memory blocks are connected through the layers. This block structure provides higher performance than the classical neuron, and adds short-term memory to the system.

The memory block receives an input sequence and each gate inside uses sigmoid activation units to control its triggering. There are three types of gates in one unit: Forget Gate, Input Gate and Output Gate. Each unit is like a small state machine in which the gates have weights that are defined during training.

The problem is in general terms a regression problem. That is, given the recorded behavior of the channels for a gesture in each of the three users, how to characterize the curves for the gesture?

The performance of the final model is evaluated using cross validation. To do this, we separate the dataset in an orderly manner, creating a training set and a test set. For training we use $67 \%$ of the data and we use the rest to test the model.

The network has a visible layer with one input, a hidden layer with eight LSTM blocks or neurons and an output layer that makes a single value prediction. The default sigmoid activation function is used for the LSTM blocks. The network is trained for 100 epochs. Performance is calculated using the same units of data.

We generate predictions of the models constructed for each channel in each of the three users. Predictions are constructed for both the train and test dataset. The visual representation is shown as follows:

- User 1: Male, ectomorph body, little hair on the arm, 20 years old Figure 4.

- User 2: Woman, endomorph body, moderate hair in the arm, 21 years old Figure 5.

- User 3: Male, endomorph body, little hair on the arm, 22 years old Figure 6.

In these figures the original dataset is in blue, the predictions for the training dataset is in green and the predictions on the unseen test dataset is in red. In each of the models the performance is calculated by evaluating the average error on the training dataset and the average error on the test dataset. The range of these values is in the same universe of discourse as the data.
Raw EMG signals were collected with Myo placed on arm's transradial portion. Data were transmitted via Bluetooth to a computer. The manipulation and analysis of the data was done in Python. The model fit code was written in Python using Keras. We setup a SciPy work environment with Pandas support. The data are originally integer values, but are converted to floating point since in this format they are more suitable for working with neural networks. LSTM networks are quite sensitive to the scale of input data due to the behavior of the activation function. Therefore, the values are rescaled to the range of 0 to 1 .

\section{Results and Discussion}

Beyond building a system of identification of gestures independent of users, we want in this research to determine the performance and reliability of a classification of gestures from similarity metrics with reference signals. A first step in this process is to have reliable and adjustable models for the sensor signals. Un segundo paso, en una investigación futura, se centrará en establecer dichos modelos, así como las métricas de similaridad. A second step, in future research, will focus on establishing such models as well as similarity metrics.

Tables 1, 2 and 3 show the performance achieved by each identified model. The performance of the models is calculated by evaluating the average error on the training dataset and the average error on the test dataset. The values shown in these tables are in the same range of values as the data, so when considering the scale, it is observed that in the worst case none of the errors exceeds $7 \%$.

We attribute the high performance of the models to the structure of the LSTM network. The general behavior of the data presents a valley and two peaks, but the values are strongly dependent on the development of the movement and therefore on the history of events. These characteristics are observed both during experiments and in the structure of the model.

\section{Conclusions}

This work has presented the development of models for signals produced by an 8-channel myoelectrical sensor, in order to construct a set of pattern signals for the recognition of gestures in a human arm. The signals were taken from three young people with different sex and body size. 

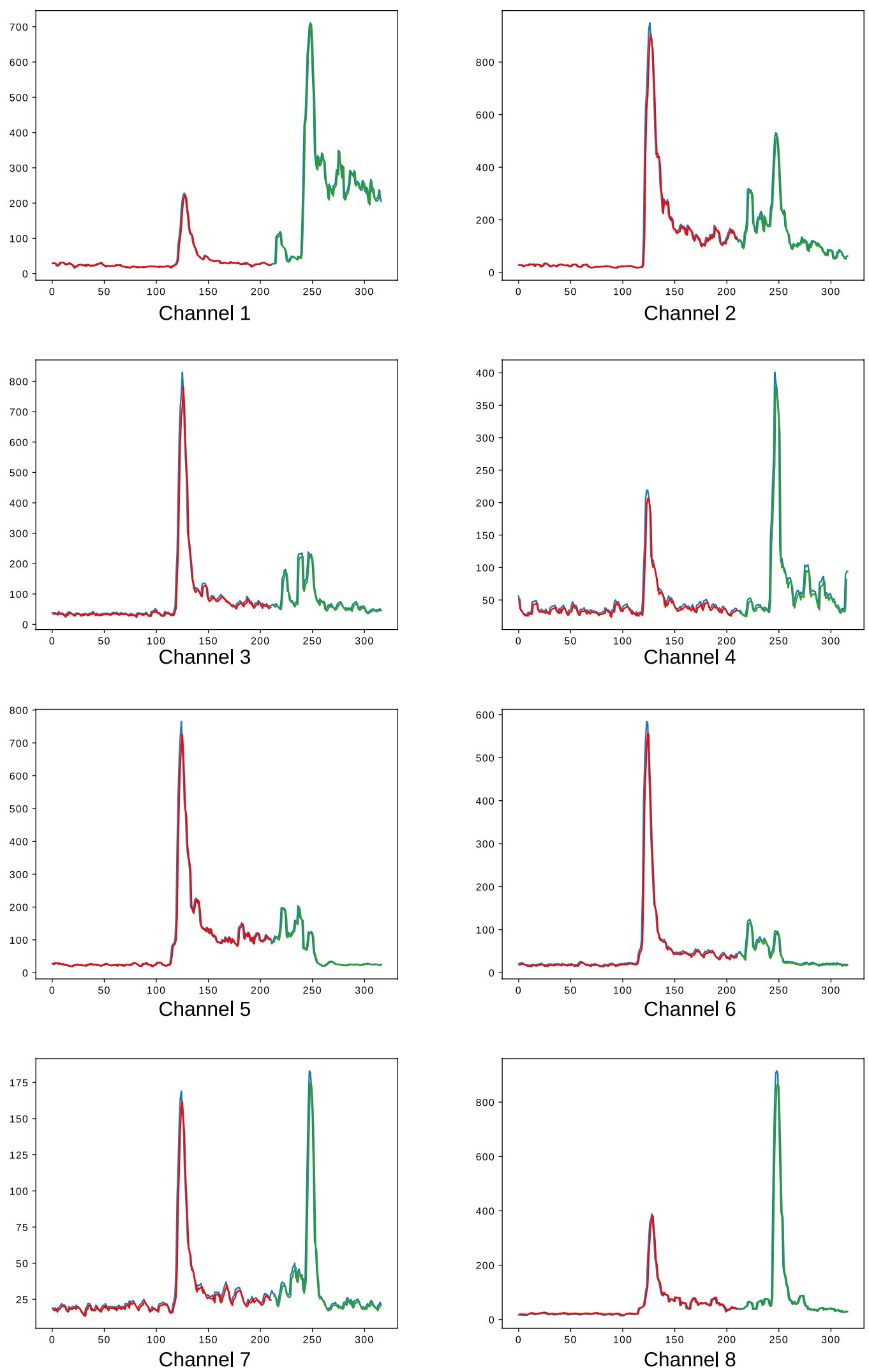

Figure 4. Eight EMG signals (blue), predictions for the training dataset (green), and predictions for the unseen test dataset (red) for User 1 (Male, ectomorph body, little hair on the arm, 20 years old). 

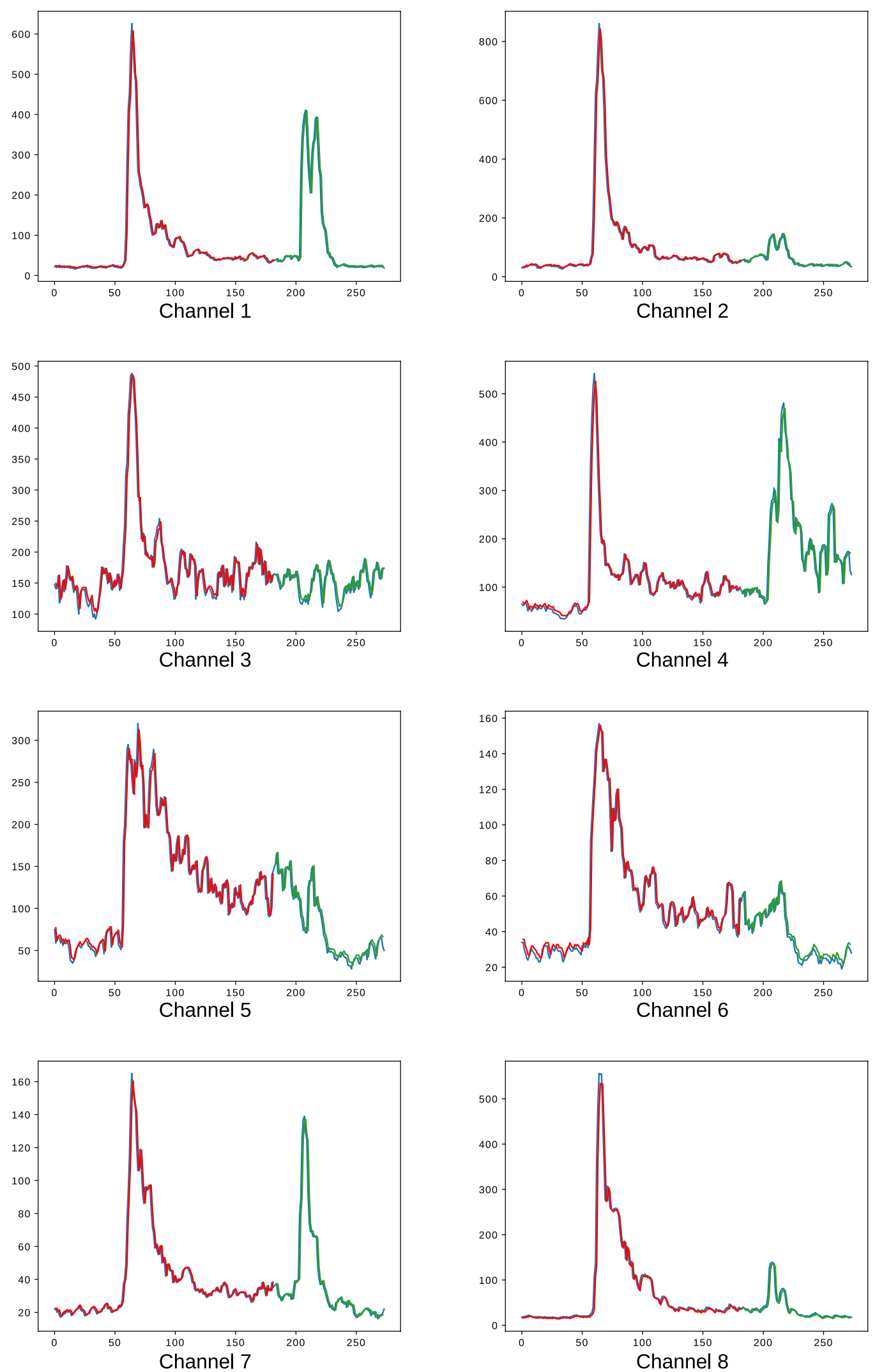

Figure 5. Eight EMG signals (blue), predictions for the training dataset (green) and predictions for the unseen test dataset (red) for User 2 (Woman, endomorph body, moderate hair in the arm, 21 years old). 

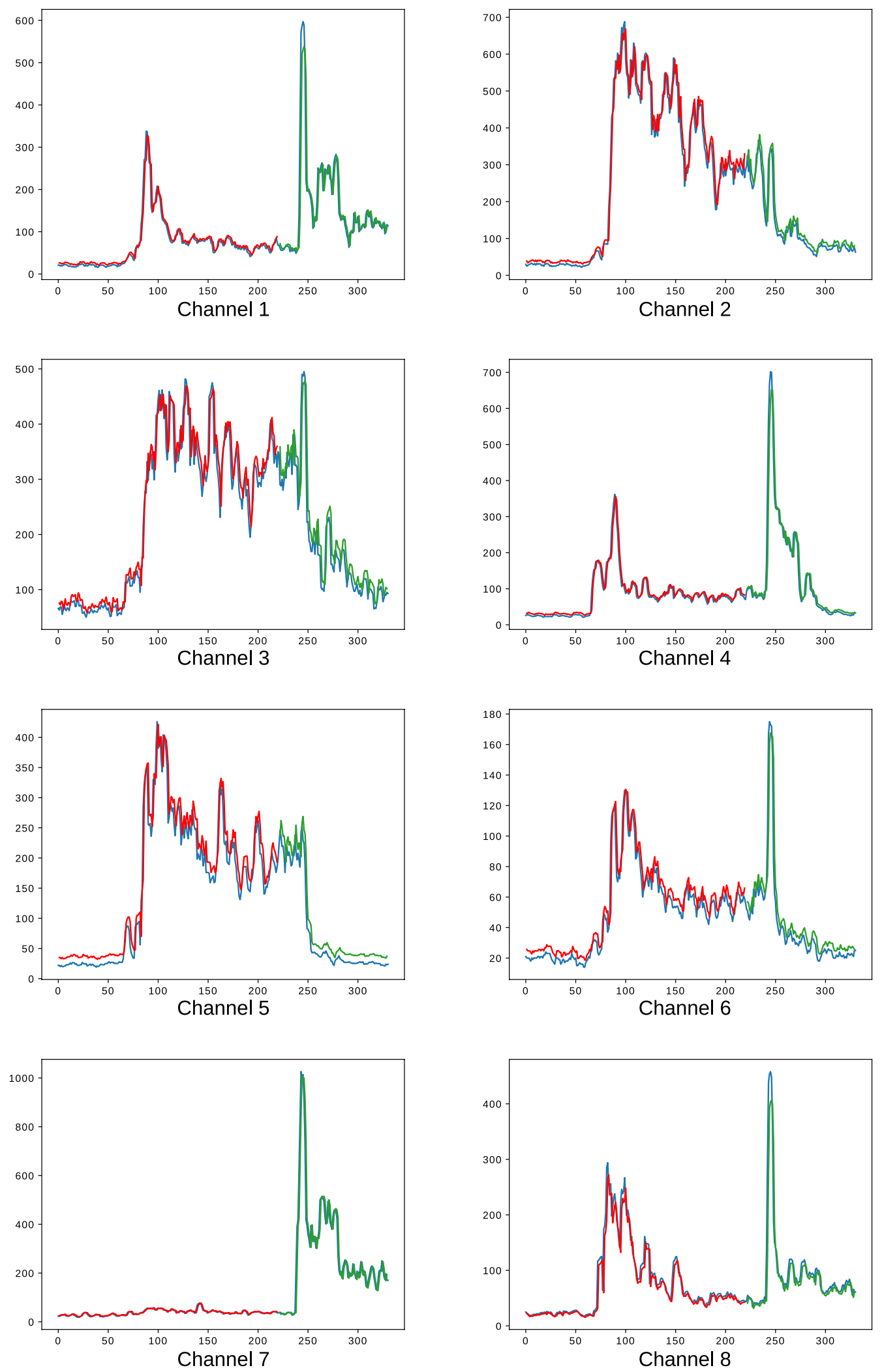

Figure 6. Eight EMG signals (blue), predictions for the training dataset (green) and predictions for the unseen test dataset (red) for User 3 (Male, endomorph body, little hair on the arm, 22 years old). 
Table 1. Performance of the model on the train and test datasets: User 1 (Male, ectomorph body, little hair on the arm, 20 years old)

\begin{tabular}{|c|l|}
\hline Channel & \multicolumn{1}{c|}{ Performance } \\
\hline 1 & $\begin{array}{l}\text { Train score: } 7.47 \text { RMSE } \\
\text { Test score: } 39.34 \text { RMSE }\end{array}$ \\
\hline 2 & $\begin{array}{l}\text { Train score: } 35.41 \text { RMSE } \\
\text { Test score: } 31.46 \text { RMSE }\end{array}$ \\
\hline 3 & $\begin{array}{l}\text { Train score: } 30.00 \text { RMSE } \\
\text { Test score: } 24.75 \text { RMSE }\end{array}$ \\
\hline 4 & $\begin{array}{l}\text { Train score: } 10.16 \text { RMSE } \\
\text { Test score: } 28.45 \text { RMSE }\end{array}$ \\
\hline 5 & $\begin{array}{l}\text { Train score: } 27.26 \text { RMSE } \\
\text { Test score: } 15.92 \text { RMSE }\end{array}$ \\
\hline 6 & $\begin{array}{l}\text { Train score: } 22.50 \text { RMSE } \\
\text { Test score: } 9.48 \text { RMSE }\end{array}$ \\
\hline 7 & $\begin{array}{l}\text { Train score: } 6.01 \text { RMSE } \\
\text { Test score: } 10.30 \text { RMSE }\end{array}$ \\
\hline 8 & $\begin{array}{l}\text { Train score: } 14.09 \text { RMSE } \\
\text { Test score: } 55.06 \text { RMSE }\end{array}$ \\
\hline
\end{tabular}

Table 2. Performance of the model on the train and test datasets: User 2 (Woman, endomorph body, moderate hair in the arm, 21 years old)

\begin{tabular}{|c|l|}
\hline Channel & \multicolumn{1}{c|}{ Performance } \\
\hline 1 & $\begin{array}{l}\text { Train score: } 24.54 \text { RMSE } \\
\text { Test score: } 32.82 \text { RMSE }\end{array}$ \\
\hline 2 & $\begin{array}{l}\text { Train score: } 32.66 \text { RMSE } \\
\text { Test score: } 9.34 \text { RMSE }\end{array}$ \\
\hline 3 & $\begin{array}{l}\text { Train score: } 20.01 \mathrm{RMSE} \\
\text { Test score: } 11.98 \text { RMSE }\end{array}$ \\
\hline 4 & $\begin{array}{l}\text { Train score: } 22.76 \text { RMSE } \\
\text { Test score: } 34.67 \text { RMSE }\end{array}$ \\
\hline 5 & $\begin{array}{l}\text { Train score: } 16.58 \text { RMSE } \\
\text { Test score: } 11.59 \text { RMSE }\end{array}$ \\
\hline 6 & $\begin{array}{l}\text { Train score: } 7.05 \text { RMSE } \\
\text { Test score: } \text { 4.28 RMSE }\end{array}$ \\
\hline 7 & $\begin{array}{l}\text { Train score: } 5.63 \text { RMSE } \\
\text { Test score: } 7.68 \text { RMSE }\end{array}$ \\
\hline 8 & $\begin{array}{l}\text { Train score: } 25.35 \text { RMSE } \\
\text { Test score: } 10.15 \text { RMSE }\end{array}$ \\
\hline
\end{tabular}

The model for each of the signals was identified using an LSTM network. This neuronal structure was selected by observing the importance of the previous events in the behavior of the signals. The network structure was designed with an input layer, a hidden layer with eight LSTM blocks or neurons, and an output layer. The per-
Table 3. Performance of the model on the train and test datasets: User 3 (Male, endomorph body, little hair on the arm, 22 years old)

\begin{tabular}{|c|l|}
\hline Channel & \multicolumn{1}{c|}{ Performance } \\
\hline 1 & $\begin{array}{l}\text { Train score: } 13.22 \text { RMSE } \\
\text { Test score: } 45.10 \text { RMSE }\end{array}$ \\
\hline 2 & $\begin{array}{l}\text { Train score: 34.22 RMSE } \\
\text { Test score: } 28.26 \text { RMSE }\end{array}$ \\
\hline 3 & $\begin{array}{l}\text { Train score: } 28.74 \text { RMSE } \\
\text { Test score: } 36.20 \text { RMSE }\end{array}$ \\
\hline 4 & $\begin{array}{l}\text { Train score: } 13.72 \text { RMSE } \\
\text { Test score: } 39.02 \text { RMSE }\end{array}$ \\
\hline 5 & $\begin{array}{l}\text { Train score: 26.18 RMSE } \\
\text { Test score: } 20.99 \text { RMSE }\end{array}$ \\
\hline 6 & $\begin{array}{l}\text { Train score: } 7.74 \text { RMSE } \\
\text { Test score: } 11.34 \text { RMSE }\end{array}$ \\
\hline 7 & $\begin{array}{l}\text { Train score: 3.78 RMSE } \\
\text { Test score: } 63.95 \text { RMSE }\end{array}$ \\
\hline 8 & $\begin{array}{l}\text { Train score: } 17.82 \text { RMSE } \\
\text { Test score: } 31.02 \text { RMSE }\end{array}$ \\
\hline
\end{tabular}

formance of the models is calculated by evaluating the average error on the training dataset and the average error on the test dataset. According to the results, the models predict the behavior of the signals faithfully, since in the worst case the error does not exceed 7\%. Consequently, this research will continue to generate models for a larger number of users, establishing baseline behavior and designing metrics for comparing similarity between the sensor signals and references in real time.

\section{Acknowledgments}

This work was supported by CIDC of District University Francisco Jose de Caldas through the project 1-73-53016 . The views expressed in this paper are not necessarily endorsed by District University. The authors thank the research groups ARMOS and DIGITI for the evaluation carried out on prototypes of ideas and strategies.

\section{References}

1. Molaei MS, Reza M. An analytical review for event prediction system on time series. 2nd International Conference on Pattern Recognition and Image Analysis; 2015. p. 1-6. https://doi.org/10.1109/PRIA.2015.7161635

2. Bobadilla L, Martinez F, Gobst E, Gossman K, LaValle S. Controlling wild mobile robots using virtual gates and 
discrete transitions. American Control Conference; 2012. p. 743-9. https://doi.org/10.1109/ACC.2012.6315569

3. Gonzalez O, Acero D. Robot semi-autonomo para el transporte de pacientes con movilidad reducida. Tekhne. 2016; 13(2):49-63.

4. Socas R, Dormido S, Dormido R. Optimal threshold setting for event-based control strategies. IEEE Access. 2017; 5(1):2880-93. https://doi.org/10.1109/ACCESS.2017. 2671419

5. Wang Y, Xing W, Zhang H. Dynamic event-based control of nonlinear stochastic systems. IEEE Transactions on Automatic Control. 2017; 62(12):6544-51.

6. Mehrmolaei S, Reza M. Time series forecasting using improved ARIMA. Artificial Intelligence and Robotics; 2016. p. 92-7. https://doi.org/10.1109/RIOS.2016.7529496

7. Shahana A, Preeja V. Survey on feature subset selection for high dimensional data. International Conference on Circuit, Power and Computing Technologies (ICCPCT 2016); 2016. p. 1-4. https://doi.org/10.1109/ICCPCT.2016.7530147

8. Sharma P, Mathur A, Chaturvedi S. An improved fast clustering-based feature subset selection algorithm for multi featured dataset. International Conference on Advances in Engineering and Technology Research (ICAETR 2014); 2014. p. 1-5. https://doi.org/10.1109/ICAETR.2014.7012880

9. Krawczak M, Szkatula G. Time series envelopes for classification. 5th IEEE International Conference Intelligent Systems (IS 2010); 2010. p. 1-6. https://doi.org/10.1109/ IS.2010.5548371

10. Sevcech J, Bielikova M. Symbolic time series representation for stream data processing. IEEE Trustcom/BigDataSE/ISPA. 2015; 2:217-22. https://doi.org/10.1109/Trustcom.2015.586

11. Nguyen L, Novak V. Filtering out high frequencies in time series using F-transform with respect to raised cosine generalized uniform fuzzy partition. IEEE International
Conference on Fuzzy Systems (FUZZ-IEEE 2015); 2015. p. 1-8. https://doi.org/10.1109/FUZZ-IEEE.2015.7337864

12. Holcapek M, Novak V, Perfilieva I. Noise reduction in time series using F-transform. IEEE International Conference on Fuzzy Systems (FUZZ 2013). 2013; p. 1-8. https://doi. org/10.1109/FUZZ-IEEE.2013.6622492

13. Qiao T, Ren J, Wang Z, Zabalza J, Sun M, Zhao H, et al. Effective denoising and classification of hyperspectral images using curvelet transform and singular spectrum analysis. IEEE Transactions on Geoscience and Remote Sensing. 2017; 55(1):119-33. https://doi.org/10.1109/TGRS.2016.2598065

14. Tran T, Kiyoshi, Yokokura Y, Xuan T, Yabuki A. High performance load acceleration control based on singular spectrum analysis for industrial robot. IEEE International Power Electronics and Motion Control Conference (PEMC 2016); 2016. p. 824-9.

15. Ayala G, Sebastian R, Diaz M, Diaz E, Zoncu R, Toomre D. Analysis of spatially and temporally overlapping events with application to image sequences. IEEE Transactions on Pattern Analysis and Machine Intelligence. 2006; 28(10): 1707-12. PMid:16986551. https://doi.org/10.1109/TPAMI. 2006.199

16. Li L, Su X, Zhang Y, Lin Y, Li Z. Trend modeling for traffic time series analysis: An Integrated Study. IEEE Transactions on Intelligent Transportation Systems. 2015; 16(6):3430-9. https://doi.org/10.1109/TITS.2015.2457240

17. Schluter T, Conrad S. About the analysis of time series with temporal association rule mining. IEEE Symposium on Computational Intelligence and Data Mining (CIDM 2011); 2011. p. 1-8. https://doi.org/10.1109/CIDM.2011.5949303

18. Hoang N, Tuan D. Comparison of strategies for multi-stepahead prediction of time series using neural network. International Conference on Advanced Computing and Applications (ACOMP 2015); 2015. p. 142-9. 